\title{
Diagnostic of craniofacial asymmetry. Literature review
}

\author{
Rosa-María Yáñez-Vico ${ }^{1}$, Alejandro Iglesias-Linares ${ }^{2}$, Daniel Torres-Lagares ${ }^{3}$, José-Luis Gutiérrez-Pérez ${ }^{4}$, \\ Enrique Solano-Reina 5
}

\author{
${ }^{1}$ Research Scholarship. Stomatology Department. Proffesor of the Orthodontics and Dentofacial Orthopeadics Master. School \\ of Dentistry. University of Seville \\ ${ }^{2}$ Proffesor of the Orthodontics and Dentofacial Orthopeadics Master. School of Dentistry. University of Seville \\ ${ }^{3}$ Proffesor of Oral surgery. Stomatology Department. University of Seville \\ ${ }^{4}$ Chairmain of Oral surgery. Stomatology Department. University of Seville. Chef of UGC in Oral and Maxillofacial Surgery. \\ HU Virgen del Rocío. Seville \\ ${ }^{5}$ Chairman of Orthodontics. Stomatology Department. University of Seville
}

Correspondence:

HU Virgen del Rocio

School of Dentistry of Seville

C/ Avicena s/n 41009

Seville. Spain

danieltl@us.es

Received: 22/03/2009

Accepted: $28 / 11 / 2009$
Yáñez-Vico RM, Iglesias-Linares A, Torres-Lagares D, Gutiérrez-Pérez JL, Solano-Reina E. Diagnostic of craniofacial asymmetry. Literature review. Med Oral Patol Oral Cir Bucal. 2010 May 1;15 (3):e494-8. http://www.medicinaoral.com/medoralfree01/v15i3/medoralv15i3p494.pdf

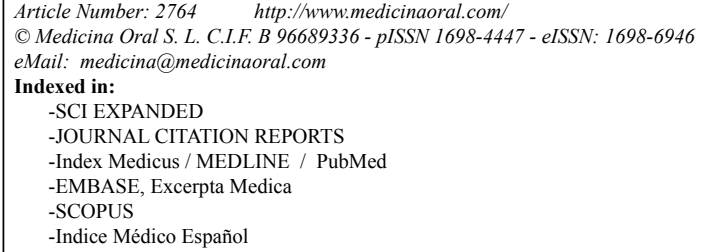

\begin{abstract}
Facial asymmetry is a common feature in many syndromes, and requires surgery as the only valid treatment option. Routine diagnostic methods (frontal RX, panoramic RX and submentovertex RX) have serious limitations mainly due to the transfer from a three dimensional image to a two dimensional plane. The feasibility of such methods is poorly supported due to inherent projection errors (image magnification, cranial rotation) and identification errors (image quality, precision and reproducibility). The use of computer tomographies represents a substantial improvement in the sense of skeletal and soft tissue structures' reproduction precision. The interpretation of this new data source makes evident the necessity of new analysis tools for extraction, manipulation and synthesization of the whole diagnostic and therapeutical potential based on more solid studies in this field.
\end{abstract}

Key words: Cranio-facial asymmetry, x ray diagnosis, cephalometry, diagnostic error.

\section{Introduction}

Facial aesthetics has exponentially increased its importance in all health fields. As a consequence, patient requirements and demands have grown in a parallel manner and require of constant professional improvements. Scientific literature has shown a great connexion between symmetry and attraction in animals and also in humans (1).
Craniofacial asymmetry is not only an aesthetic aspect but also a feature of pathology due to inherent symmetry on many skeletal structures. Additionally a substantial number of dysmorphic syndromes are related to craniofacial anomalies and severe facial asymmetries. Structure variability, malformative range, malformative aetiology and individual reaction are characteristic features 
of these malformative syndromes. Many orthogenetic surgery patients have asymmetrical facial characteristics after the surgical procedure and in many cases they only pay attention to them after the surgery. Therefore, a correct diagnostic is necessary in order to perform an optimum and efficient therapeutic approach.

Vig et al. (2) evaluated 63 frontal radiographies of children between 9-18 years of age to determine symmetry in different parts of the face. The children didn't show clinical asymmetrical characteristics. Surprisingly, a majority of the children showed a general asymmetrical pattern. According to Vig et al. the left side of the face was the longest part. The dentoalveolar region was the most symmetrical region probably caused by compensatory changes due to dentoalveolar development that allowed a symmetrical masticatory function and a maximum intercuspidation. The border between pathological and normal asymmetry is not as clear as it should be. Usually this concept is related to the clinical opinion and to the own perception of the patient. Due to the significant improvements that have occurred in the field of Medicine and Informatics, the majority of the classifications used nowadays for asymmetry or craniofacial deformities have become obsolete. The importance of specific cranial growth points must be highlighted along with its role in these concrete malformative syndromes.

In sum, a diagnostic error in the craniofacial asymmetry due to a misinterpretation or a poor identification of the structures implicated on the asymmetry could limit the therapeutical options leading to an erroneous treatment. A review of the current literature in the field is present in this article. This article, as well, compiles diagnostic material and methods routinely used for the diagnostic of the asymmetry.

\section{Panoramic Radiography}

Panoramic X-ray technology is commonly accessible and used in daily clinical routine. These radiographies allow a bilateral view and are adequate to inform on vertical measurements. This is the main reason for using them for asymmetry evaluation of the condylar and the ramus process and for measuring vertical differences between both sides. In this way Habets et al. (3) (Fig. 1), Sağlam et al. (4) (Fig. 2) methods must be highlighted among others.

Since the 80's, different authors (5) have explored this diagnostic tool for measuring ramus and condyle vertical differences. These authors conclude that many deficiencies must be the result of the distortion and magnification of the ramus and the condyle. Additionally the condyle structure is frequently superimposed with the lateral edge of the glenoid fossa and zygomatic arch root. Although panoramic x-ray technology has an acceptable cost-benefit ratio due to the minimum radiation

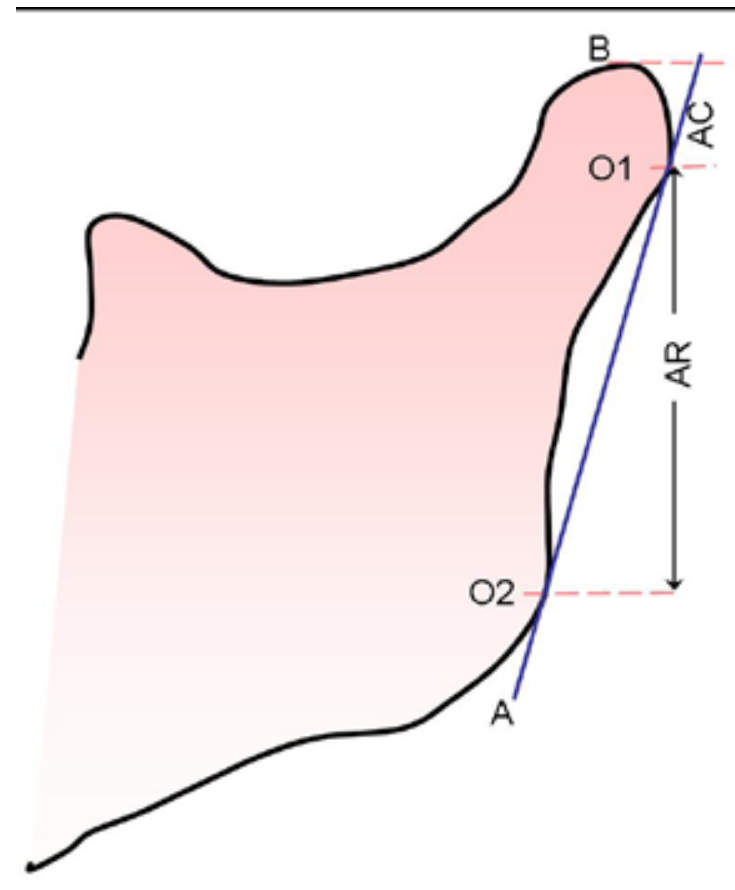

Fig. 1. Habets et al method (1988). AC: condylar process height; AR: mandibular ramus height; A: tangent to the mandibular ramus; $\mathrm{B}$ : tangent to the condyle and perpendicular to $\mathrm{A} ; \mathrm{O} 1$ y O2: lateral points of the condyle and mandibular ramus, respectively.

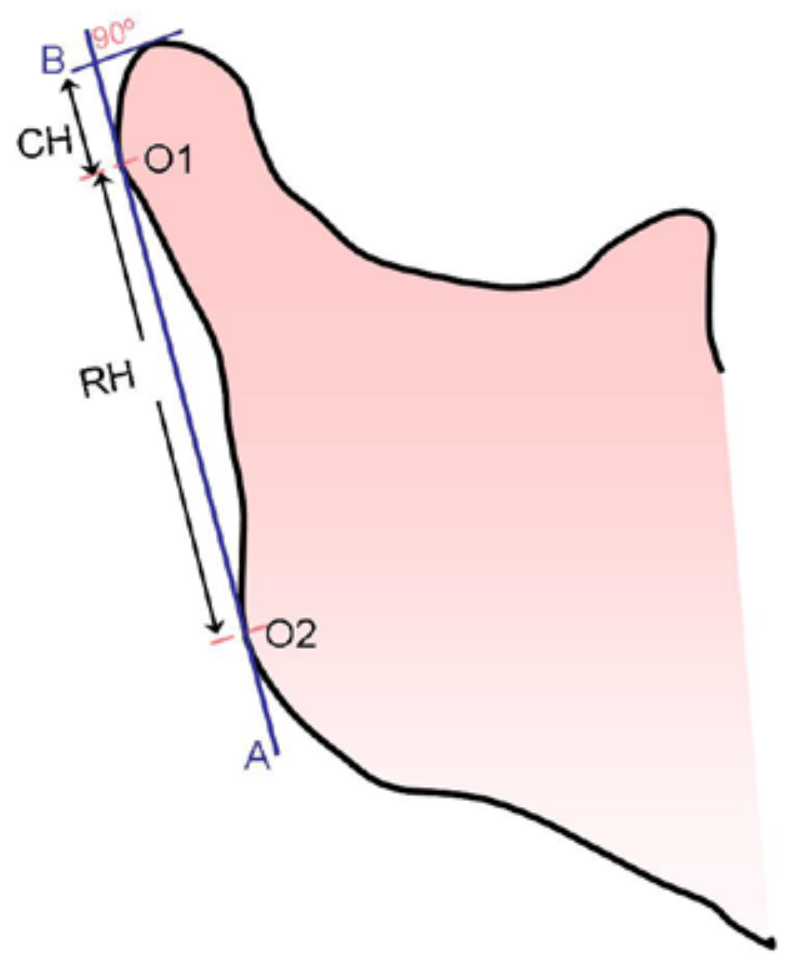

Fig. 2. Saglam and Santi method(2004). A: tangent to the ramus; B: perpendicular to $\mathrm{A}$; $\mathrm{CH}$ condylar height: $\mathrm{RH}$ ramus height; $\mathrm{O} 1$ y $\mathrm{O} 2$ the most lateral points. 
exposure, the literature doesn't confer big trust to measurements done with this type of projection. This method is susceptible to distortion and movement of the image due to a tight image in the anterior area (6). There are even some authors such as Ramstad et al. (6) that support the necessity of avoiding any kind of measurement performed on panoramic radiographs.

However, other authors think that standard head positioning and bitten wax diminish the majority of distortions (4). Due to the nonlinear variation that occurs by the different depths there is no controversy on the invalidity of the horizontal measurements. Therefore, Larheim et al. (7) thinks that vertical and angular measurements can be reproduced if the patient is provided with adequate equipment for head positioning. Recently a systematic review (8) has highlighted once more the poor feasibility of measurements performed on this kind of x-ray and the high variability depending on the $\mathrm{x}$-ray machine.

\section{Posteror/Anterior Radiography}

Despite the fact that the posterior radiography has become one of the most common clinical x-ray projections for the diagnostic of asymmetry, it's generally assumed in the literature that it is especially difficult to perform valid measurements for such purpose (9). There are many studies that evaluate the validity of the frontal cephalometry $(10,11)$. These studies conclude that there are two basic errors in the posterior/anterior projections: those related to the cephalometric method (object-film distance, head rotation) and those inherent to the method (reference points localization, identification reference points due to structures radiolucency, structure superimpositions) (12).

Referring to the head positioning, a simple head rotation would be enough to disturb the perpendicularity of the cranial mediosagital line of the x-ray beam leading to distorted measurements. Even if ideal positioning were achieved, the location of a proper mediosagital axis in those patients affected of severe asymmetries still becomes especially complicated. Furthermore, we often consider a proper perpendicular positioning of the connection axis to be between both external auditory holes and the mediosagital line, which is usually completely inaccurate. Therefore, asymmetric ears would provoque a head rotation and therefore a misinterpretation of the asymmetry (13).

Usually cephalometric errors extracted from the projection of a three dimensional head in a two dimensional x-ray film are not taken into account. Accordingly, Chidiac et al. (13) evaluated those errors. The rate of cephalometric distortion decreases gradually the closer the structures are to the x-ray film. It also differs depending on different planes; mandibular width (defined as the distance between right antegonial and left ante- gonial: AG-GA, being antegonial $-\mathrm{AG}-$ the medialest point in the antegonial notch of the mandible) was a value of $4,42 \%$ and the maxilar width (right jugal -left jugal: JL-JR, being jugal-J- the most inferior point of the zygomatic-alveolar crest) was a value of $1,83 \%$, if the film was situated $13 \mathrm{~cm}$ away of the transporionic axis (line that connects both external auditory holes). This fact suggests that the diagnostic of the difference between the maxilar and the mandibular width increases the AG-GA value difference by approximately $2,5 \%$. Concerning the precision in the location of the reference points, different authors $(13,14)$ agree on the necessity of using the reference points with a variation of less than $1,5 \mathrm{~mm}$ for the cephalometric analyses. Mayor et al. (14) outlined $2 \mathrm{~mm}$ as the vertical localization error of the point $\mathrm{Cr}$ (Galli Crest: the superior point of the galli crest of the ethmoidal bone). The literature (14) recommends a critical position on the validation of the maxilar and mandibular width.

The $\mathrm{J}$ point experiences a variation of $2,60 \mathrm{~mm}$ in a horizontal direction and $3,06 \mathrm{~mm}$ in a vertical direction. However, usually in the clinical practice, the transversal analysis is limited to the difference in between both jaws, what seriously limits the treatment options and the treatment planning.

\section{Submentovertex Projection}

This kind of projection is more useful than the other two cited due to the excellent visualization of the cranial base structures that allow the use of the anatomical reference points over the cranial base to determine the mediosagital axis. Despite its inherent benefits, it's used much less in the clinical practice than those described above.

Berger was the first author that supported the usefulness of the submentovertex projection (SMV) for the evaluation of asymmetry in cephalometry. Gilbert investigated the precision of this kind of projection with a film orientated parallel to the horizontal plane of Frankfort. The width factors were found highly reproducible. Nevertheless, significant longitudinal measurement errors were found. This author didn't suggest any definitive cephalometric analyses. Ritucci and Burnstone used reference points on the cranial base and they developed a cephalometric analysis for the evaluation of the asymmetry on the craniofacial complex. However, Foresberg et al in 1984 (Fig. 3) and Grayson et al in 1985 were the first authors to publish a method for the evaluation SMV $\mathrm{x}$-rays using a multi-planning skeletal tracing analyses on the cranial base, midface and lower part of the face. This method allowed the calculation of asymmetry on the cranial base, the zygomatic complex and the mandible. All reference points calculated related to a coordinate system from the spinous foramen, the most stable and reproducible point in the cranial base.

Williamson et al. (15) studied the identification errors 


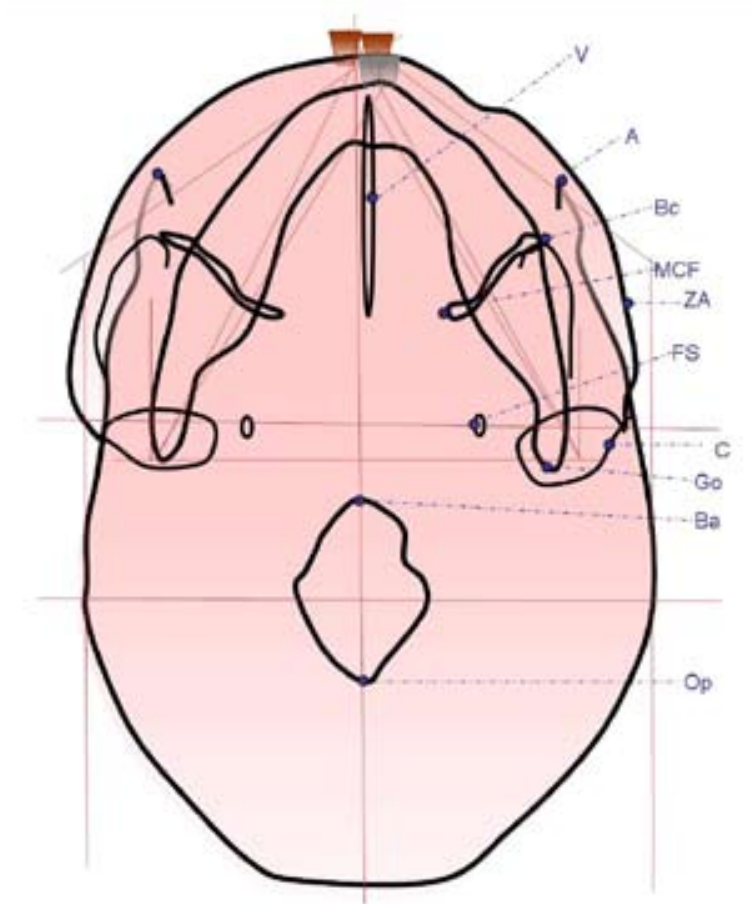

Fig. 3. Foresberg et al method (1984). A: angular; BC: bucal; MCF: medial cranial fosse; ZA: zygomatic arch; V: vomer; C: condyle; Go: gonion; FS: spinous foramen; Ba: basion; Op: opistion.

in SMV x-ray. The most significant discrepancy was found in the pogonion (Pg: the most anterior point on the mandible in the medial sagital plane of the mandibular symphysis), up to $3,79 \mathrm{~mm}$. Additionally, a tendency of superimposition of the internal and the external corticals of the frontal and the dentition over the anterior region of the mandibular symphysis was found. This superimposition is substantially influenced by head positioning. Lysell et al. (16) suggested that the projection effects would be minimal if the mandibular angle were projected immediately before to the condyle. The ideal positioning of the head for the SMV radiographies would be that in which the X-ray beam is perpendicular to the Frankfort plane; however, several limitations exist in patients with reduced mobility of the neck. The intra and interexaminator errors were of low reliability in the condylar poles, especially in the vertical direction and in the posterior condylar point of the horizontal direction. Related to that fact, the authors suggest being cautious in the interpretation and use of the measurements that involve such points due to the high number of errors tempted with this method.

\section{From the two to the three dimensions: The com- puterized tomography}

The arrival of the digital image to the dental world has provoked new research fields focused on the diagnostic potential of the radiography through image manipulation. Many of those proposals have resulted in valuable tools that increase the diagnostic utility. However many digital images suffer from limitations similar to those derived from the conventional cephalometric analyses including magnification, distortion and superimposition of anatomical structures.

With the arrival of the computerized tomography the totality of the craniofacial complex can be analyzed with high precision. The promoters of computerized tomography (CT) were Cormack and Hounsfield during the 60's-70's. These authors were given the Nobel Prize of Medicine. This technology has promoted new tools for medical research and has become one of the most extensive in the image diagnostic methods nowadays. The CT has proportioned the capacity to visualize specific tissues in several sequential planes without the problem of superimposition. It is a fact that the computerized tomography has become established simultaneously with the improvement of the scanners used.

An important advancement in the CT technology has come thanks to Herman and Liu. In 1977 these authors introduced three dimensional reconstructions from axial slides. This method did away with the necessity of mental assimilation of three dimensional images from two dimensional data (those from conventional $\mathrm{x}$-ray machines and from axial CT scanners), which is often inaccurate to impossible.

The CT allows us an exact determination of the anatomical structures in three dimensions, thus providing us with an improvement in the preparatory planning of many surgical procedures (17). The CT allows for the visualization of both the soft tissues and the skeletal structures in 3D. The majority of the literature demonstrates the high precision of the CT-3D images (18). Moreover, there is no superimposition of anatomical structures and an absolute accurate position of the anatomical reference points can be obtained from all angles. Unfortunately, the effective dose that is provided by a CT medical scanner is higher than that obtained from the conventional x-ray. Furthermore, the CT is relatively more expensive and the scanners are not easily accessible.

A new generation of compact $\mathrm{CT}$ scanners has been developed, specially designed for use in the head and neck region. Those especially compact $\mathrm{CT}$ scanners use the cone beam geometry (cone beam - CBCT) that allows for a high efficiency in uses with x-ray photons. The dose provided by the CBCT is relatively low and even lower than that received by a periapical complete series and 100 times lower than that given by a medical CT scanner. The reconstruction of the CBCT offers a broad field of vision $(22-30 \mathrm{~cm})$, allowing the reconstruction and three dimensional visualization of the maxillofacial structures. Additionally, conventional images as panoramic, lateral and anterior/posterior radiographs can be obtained from those CBCT.

The substitution of the conventional $\mathrm{x}$-ray machines by 
the CBCT for the study of the craniofacial structures is a potential advancement in the treatment and diagnosis for the wide range of orthodontic and orthogenetic patients (19). The CBCT allows for the reduction of many limitations extracted from conventional radiographies. Nevertheless, three dimensional images mean new changes and the need for a new interpretation from that which we are used to for extracting the most information possible.

Many techniques have been used in the CT reconstruction for the diagnostic, planification and simulation of the treatment. However, many changes on the superimposition of images are now appearing for the monitoring of changes related to the treatment of the structures. Three dimensional reference point identification requires new functional definitions suitable for the three dimensions of space (20). The uses of a precise and consistent instrument for the analysis of a wide range of images obtained from this kind of technology, sets out new diagnostic possibilities for medical practitioners.

\section{References}

1. Enquist M, Arak A. Symmetry, beauty and evolution. Nature. 1994;372:169-72.

2. Vig PS, Hewitt AB. Asymmetry of the human facial skeleton. Angle Orthod. 1975;45:125-9.

3. Habets LL, Bezuur JN, Naeiji M, Hansson TL. The Orthopantomogram, an aid in diagnosis of temporomandibular joint problems. II. The vertical symmetry. J Oral Rehabil. 1988;15:465-71.

4. Saglam AA, Sanli G. Condylar asymmetry measurements in patients with temporomandibular disorders. J Contemp Dent Pract. 2004;5:59-65.

5. Habets LL, Bezuur JN, van Ooij CP, Hansson TL. The orthopantomogram, an aid in diagnosis of temporomandibular joint problems. I. The factor of vertical magnification. J Oral Rehabil. 1987;14:475-80. 6. Ramstad T, Hensten-Pettersen O, Mohn E, Ibrahim SI. A methodological study of errors in vertical measurements of edentulous ridge height on orthopantomographic radiograms. J Oral Rehabil. 1978;5:403-12.

7. Larheim TA, Svanaes DB, Johannessen S. Reproducibility of radiographs with the orthopantomograph 5: tooth-length assessment. Oral Surg Oral Med Oral Pathol. 1984;58:736-41.

8. Van Elslande DC, Russett SJ, Major PW, Flores-Mir C. Mandibular asymmetry diagnosis with panoramic imaging. Am J Orthod Dentofacial Orthop. 2008;134:183-92.

9. Ahlqvist J, Eliasson S, Welander U. The effect of projection errors on angular measurements in cephalometry. Eur $\mathrm{J}$ Orthod. 1988;10:353-61.

10. Trpkova B, Prasad NG, Lam EW, Raboud D, Glover KE, Major PW. Assessment of facial asymmetries from posteroanterior cephalograms: validity of reference lines. Am J Orthod Dentofacial Orthop. 2003;123:512-20.

11. Legrell PE, Nyquist H, Isberg A. Validity of identification of gonion and antegonion in frontal cephalograms. Angle Orthod. 2000;70:157-64.

12. Cavalcanti MG, Rocha SS, Vannier MW. Craniofacial measurements based on 3D-CT volume rendering: implications for clinical applications. Dentomaxillofac Radiol. 2004;33:170-6.

13. Chidiac JJ, Shofer FS, Al-Kutoub A, Laster LL, Ghafari J. Comparison of CT scanograms and cephalometric radiographs in craniofacial imaging. Orthod Craniofac Res. 2002;5:104-13.

14. Major PW, Johnson DE, Hesse KL, Glover KE. Landmark identification error in posterior anterior cephalometrics. Angle Orthod. 1994;64:447-54.
15. Williamson PC, Major PW, Nebbe B, Glover KE. Landmark identification error in submentovertex cephalometrics. A computerized method for determining the condylar long axis. Oral Surg Oral Med Oral Pathol Oral Radiol Endod. 1998;86:360-9.

16. Lysell L, Petersson A. The submento-vertex projection in radiography of the temporomandibular joint. Dentomaxillofac Radiol. 1980;9:11-7.

17. Harrell WE Jr, Hatcher DC, Bolt RL. In search of anatomic truth: 3-dimensional digital modeling and the future of orthodontics. Am J Orthod Dentofacial Orthop. 2002;122:325-30.

18. Cavalcanti MG, Haller JW, Vannier MW. Three-dimensional computed tomography landmark measurement in craniofacial surgical planning: experimental validation in vitro. J Oral Maxillofac Surg. 1999;57:690-4.

19. Madrigal C, Ortega R, Meniz C, López-Quiles J. Study of available bone for interforaminal implant treatment using cone-beam computed tomography. Med Oral Patol Oral Cir Bucal. 2008;13:E30712 .

20. Netherway DJ, Abbott AH, Gulamhuseinwala N, McGlaughlin $\mathrm{KL}$, Anderson PJ, Townsend GC, et al. Three-dimensional computed tomography cephalometry of plagiocephaly: asymmetry and shape analysis. Cleft Palate Craniofac J. 2006;43:201-10. 